\title{
Determining the order of chiral phase transition in QCD from conformal bootstrap
}

\author{
Yu Nakayama* \\ Walter Burke Institute for Theoretical Physics \\ California Institute of Technology, Pasadena, California 91125, USA \\ E-mail: nakayama@theory.caltech.edu
}

There has been a long-standing debate if the chiral phase transition in two-flavor massless QCD is first order or second order. The previous arguments based on epsilon expansions, large $\mathrm{N}$ expansions, functional renormalization group, and Monte-Carlo simulations had been all inconclusive with shortcomings. If it were the second order phase transition, there should exist a corresponding three-dimensional conformal field theory which describes the critical phenomenon. The recent development in conformal bootstrap enables us to directly study the (non-)existence of conformal fixed points in a non-perturbative manner. In this proceeding, I review the conformal bootstrap method and its application to this problem. Our conclusion is that the corresponding conformal fixed point should exist and the phase transition will be the second order if the U(1) chiral anomaly is effectively restored. This means that the original 1-loop prediction by Pisarski and Wilczek would be incorrect. We further provide the most precise prediction of the critical exponent there. We believe future numerical simulations will confirm our prediction.

The 33rd International Symposium on Lattice Field Theory

14 -18 July 2015

Kobe International Conference Center, Kobe, Japan*

${ }^{*}$ Speaker. 


\section{Introduction}

One of the greatest challenges to theoretical physicists is to understand the universal nature of phase transitions and critical phenomena. A significant triumph of the last century in this direction is the introduction of renormalization group (RG), which enables us to explain the infrared behaviors of physical systems from a small number of relevant parameters (see e.g. [1][2]). If a system undergoes a continuous phase transition, its critical behavior is governed by the endpoint of the RG flow realized as a scale invariant and, in most cases, conformal invariant field theory. Whether such a conformal field theory (CFT) with a given symmetry of the system exists or not is of theoretical interest because without its existence the continuous phase transition cannot occur.

In particular, there has been a huge controversy if the chiral phase transition of the QCD (with two massless quarks to make the question mathematically well-defined) is first order or second order. From this perspective, (non-)existence of fixed points in $O(n) \times O(m)$-symmetric LandauGinzburg-Wilson (LGW) Hamiltonian in $d=3$ spatial dimensions

$$
\begin{aligned}
\mathscr{H} & =\left(\partial_{\mu} \phi_{a}^{\alpha}\right)\left(\partial_{\mu} \phi_{a}^{\alpha}\right) \\
& +u\left(\phi_{a}^{\alpha} \phi_{a}^{\alpha}\right)^{2}+v\left(\phi_{a}^{\alpha} \phi_{b}^{\alpha} \phi_{a}^{\beta} \phi_{b}^{\beta}-\phi_{a}^{\alpha} \phi_{a}^{\alpha} \phi_{b}^{\beta} \phi_{b}^{\beta}\right),
\end{aligned}
$$

where $a=1, \cdots n$ and $\alpha=1 \cdots m$, plays a crucial role. In two-flavor QCD with the axial anomaly suppressed, the chiral phase transition is described by 1.1 with $v<0$ with $O(4) \times O(2) \simeq S U(2)_{L} \times$ $S U(2)_{R} \times U(1)_{A}$ symmetry. The possibility for the effective restoration of $U(1)_{A}$ symmetry at the chiral phase transition temperature has attracted a renewed interest. See e.g. [3] [4] [5] [6] [7] [8] and reference therein for recent discussions.

Despite their importance, theoretical studies of LGW models 1.1 have been notoriously hard and there remain long-standing controversies regarding what kind of fixed points actually exist. In [9], they derived the perturbative series directly at the physical dimension $d=3$ in massive-zeromomentum (MZM) scheme up to six-loop order, and in [10] minimal subtraction ( $\overline{\mathrm{MS}})$ scheme up to five-loop order. After careful resummations, the presence of non-trivial fixed points distinct from the Heisenberg ones $(v=0)$ were predicted for $n=2,3,4$ in [9][11] [12] [7], but some of the results have been criticized e.g. in [13] due to the lack of confirmation in the weak-coupling regime and the parameter dependence in the resummation. On the other hand, the functional RG truncated beyond the local potential approximation has predicted the absence of these fixed points for $n=2,3$ [14] [15]. Meanwhile, the situations in lattice Monte-Carlo simulations and experimental results have been equivocal.

Recently intensive efforts have been made toward non-perturbative understanding of higher dimensional CFTs via the conformal bootstrap program. The output of the program is (within controllable numerical errors) rigorous bounds on scaling dimensions of operators [16] [17] or operator product expansion coefficients [18] including various central charges [19] 20] [21]. One eminent feature is the presence of singular behaviors called "kinks" in the bounds and the agreement in their positions with previously known interacting CFTs, e.g. Wilson-Fisher fixed points [17] [22] 23] [24] and $d=3 O(N)$ Heisenberg fixed points [25]. Even without a firm proof, experimental success is convincing enough that the existence of the kink indicates the signal of interacting CFTs sitting there. 
In this proceeding, we report our conformal bootstrap program for $O(n) \times O(2)$-symmetric CFTs with $n=4$ [26] as a natural continuation of our previous work [27]. There we identified all the conjectured fixed points of $O(n) \times O(3)$ symmetric CFTs with sufficiently large $n$ and proposed the edge of the conformal window for the anti-chiral fixed point. Encouraged by these results, we have tackled the controversies to address the (non)-existence of conjectured CFTs. We have found a non-perturbative support for the conclusion of [9]11]- i.e., the presence of new universality classes that are distinct from Heisenberg ones.

\section{Notations and a quick summary of the $O(n) \times O(3)$-bootstrap results}

Here we briefly summarize the notations and results of our previous work [27]. We assume the presence of scalar operator $\phi_{a}^{\alpha}$ (i.e. the elementary field in 1.1) with conformal dimension $\Delta_{\phi}$ in a bifundamental representation of $O(n) \times O(m)$. The conformal block decomposition of their four-point function has nine independent channels corresponding to the irreducible representations contained in the bifundamental $\times$ bifundamental tensor product, which we label as SS, ST, SA, TS, $\cdots$, AA. Here S, T, A denote a singlet, a traceless-symmetric tensor, and an antisymmetric tensor representation of $O(n)$ and $O(m)$ symmetry group, respectively. For $O(2)$, A and $\mathrm{S}$ are distinguished by the spin of the operator.

As was first studied in [21], we can numerically compute the upper bounds on the dimension of the first operator with definite spin (labelled as $l$ ) for each sector of the representation (labelled as $R$ ) which we denote by $\Delta_{c}^{R, l}\left(\Delta_{\phi}\right)$. The upshot of [27] is that for $m=3$ and $n \gg 3$, we can identify all the fixed points as singular behaviors of the output $\Delta_{c}^{R, l}\left(\Delta_{\phi}\right)$. In other words, we can "solve" these fixed points in the same sense as in [22] [24]. In addition to the $O(3 n)$ Heisenberg fixed point, we found an unstable fixed point (called "anti-chiral" in the literature) in $\Delta_{c}^{\mathrm{TA}, 1}$ and $\Delta_{c}^{\mathrm{ST}, 0}$, or a stable fixed point (called "chiral" in the literature) in $\Delta_{c}^{\mathrm{TS}, 0}$ but did not find any interesting behaviors in the other sectors.

Below we compute the bounds for dimensions of various operators in $O(n) \times O(2)$ symmetric CFTs with 4 [26], following the scheme of [25]. We use the hybrid method of Zamolodchikov-type recursion introduced there and extremely convenient $\rho$-series expansion derived in [28] to generate partial fractional approximations for conformal blocks. Our sdpa-gmp [29] [30] implementation will be identical to the one in [27]. We do not assume intermediate scalar operator dimensions to be greater than 1 unlike in [25] [24], which is obligatory for our purpose because some of the conjectured fixed points have intermediate scalar operators with dimension below 1 .

\section{3. $n=4$ : QCD and Collinear fixed point}

In [33] it was pointed out that $S U(2)_{L} \times S U(2)_{R}$-symmetric LGW models will describe the QCD chiral phase transition which occurred in our early universe, where the bifundamental scalar fields are identified with the meson fields. As was already discussed there, the presence of anomalous $U(1)_{A}$ symmetry which may be restored by finite-temperature effects could significantly alter the conclusion. The anomaly term makes a certain meson field (including $\eta^{\prime}$ ) massive so that the resultant fixed point by tuning the temperature could be only $O(4)$ Heisenberg one (or first order). See [34]35] for the RG analysis. We point out that some recent numerical as well 
as theoretical studies suggest the possibility of the effective restoration of $U(1)_{A}$ at the transition temperature, see e.g. [3] [4] [5] [6] [7. If this is the case, the LGW model to be studied is $S U(2)_{L} \times S U(2)_{R} \times U(1)_{A} \simeq O(4) \times O(2)$ as in 1.1 for which the existence of IR-stable fixed point is again controversial. The higher order perturbative study predicts the chiral $(v>0)$ and collinear $(v<0)$ fixed points while functional RG analysis provides no evidence for them [39] [40] [41]. Note that the collinear fixed point is the one relevant in the chiral phase transition, where the symmetry breaking pattern is $S U(2)_{L} \times S U(2)_{R} \times U(1)_{A} \rightarrow S U(2)_{\text {diag. }}$. For the lattice simulations to determine the order of the chiral phase transition with conflicting results, we refer to [42] [43] [44] [45] [46].

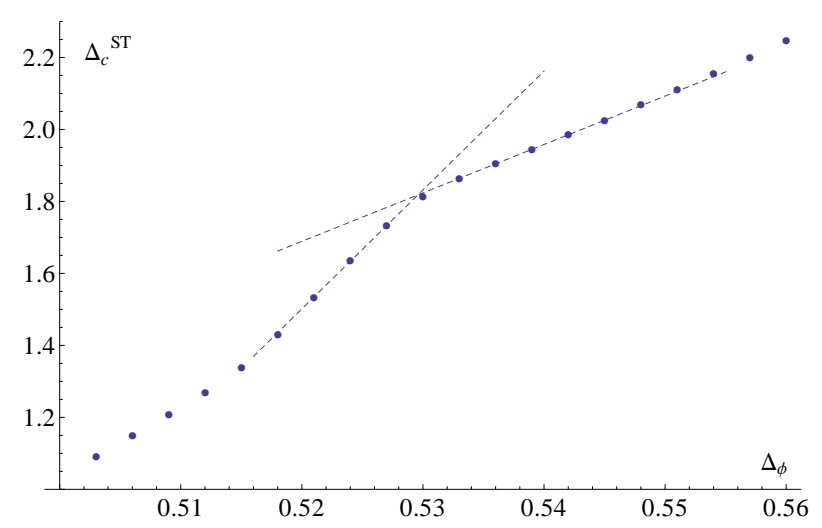

Figure 1: The bound $\Delta_{c}^{\mathrm{ST}, 0}$ for $O(4) \times O(2)$ symmetric CFTs. Here the search space dimension is $55 \times 9$, i.e., $k=10$ in the notation of [25].

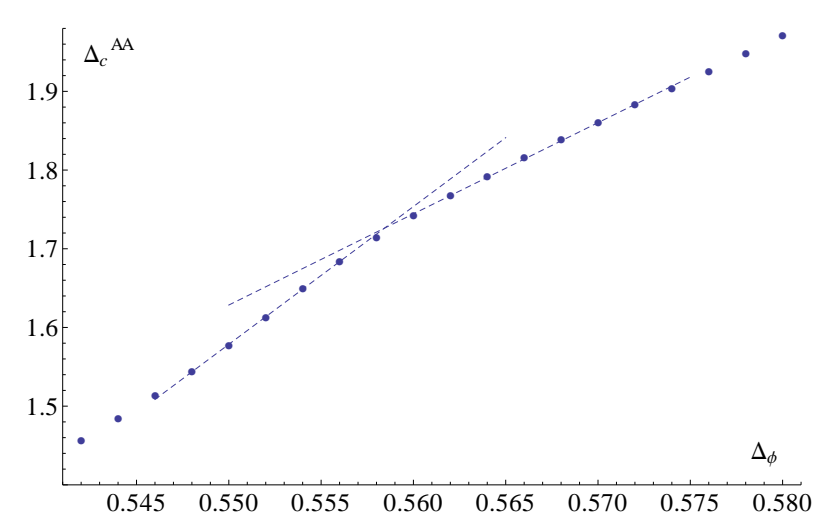

Figure 2: The bound $\Delta_{c}^{\mathrm{AA}, 0}$ for $O(4) \times O(2)$ symmetric CFTs. Here the search space dimension is $78 \times 9$, i.e., $k=12$ in the notation of [25].

We present our results for $\Delta_{c}^{\mathrm{ST}, 0}$ and $\Delta_{c}^{\mathrm{AA}, 0}$ in FIG 1 and 2 One technical remark here is that the anti-symmetric tensor representation of $O(4)$ is actually a direct sum of two irreducible representations, but we did not take this into account since the LGW model 1.1 relevant for us has a $\mathbb{Z}_{2}$-symmetry which permutes $S U(2)_{L}$ and $S U(2)_{R}$. As was pointed out in [32], one can read off 


\begin{tabular}{|c||c|c|c|c|c|c|}
\hline & $\Delta_{\phi}$ & $\Delta_{\mathrm{SS}}$ & $\Delta_{\mathrm{ST}}$ & $\Delta_{\mathrm{TS}}$ & $\Delta_{\mathrm{TT}}$ & $\Delta_{\mathrm{AA}}$ \\
\hline \hline bootstrap & $0.530(3)$ & $1.35(4)$ & $1.80(6)$ & $1.31(2)$ & $1.085(3)$ & $0.90(1)$ \\
\hline$\overline{\mathrm{MS}}$ & $0.536(5)$ & $1.44(10)$ & $1.83(8)$ & $1.35(3)$ & $1.06(10)$ & $0.83(10)$ \\
\hline MZM & $0.533(3)$ & $1.04(12)$ & $1.94(7)$ & $1.36(5)$ & $0.96(20)$ & $0.71(8)$ \\
\hline
\end{tabular}

Table 1: The low-lying spectra read off around the kink in FIG. 1 and the spectra for the $O(4) \times O(2)$ chiral fixed point from [36, 12] and 31].

\begin{tabular}{|c||c|c|c|c|c|c|}
\hline & $\Delta_{\phi}$ & $\Delta_{\mathrm{SS}}$ & $\Delta_{\mathrm{ST}}$ & $\Delta_{\mathrm{TS}}$ & $\Delta_{\mathrm{TT}}$ & $\Delta_{\mathrm{AA}}$ \\
\hline \hline bootstrap & $0.558(4)$ & $1.52(5)$ & $0.82(2)$ & $1.045(3)$ & $1.26(1)$ & $1.71(6)$ \\
\hline$\overline{\mathrm{MS}}$ & $0.56(3)$ & $1.68(17)$ & $1.0(3)$ & $1.10(15)$ & $1.35(10)$ & $1.9(1)$ \\
\hline $\mathrm{MZM}$ & $0.56(1)$ & $1.59(14)$ & $0.95(15)$ & $1.25(10)$ & $1.34(5)$ & $1.90(15)$ \\
\hline
\end{tabular}

Table 2: The low-lying spectra read off around the kink in FIG. 2 and the spectra for the $O(4) \times O(2)$ collinear fixed point from [7], 12] and [31].

the spectra contained in $\phi \times \phi$ OPE. In TABLE 1 and 2 we list the spectra at the kink $\left(\Delta_{\phi} \simeq 0.530\right.$ for $\Delta_{c}^{\mathrm{ST}, 0}$ and $\Delta_{\phi} \simeq 0.558$ for $\left.\Delta_{c}^{\mathrm{AA}, 0}\right)$. From this comparison we find it reasonable to regard the kink in $\Delta_{c}^{\mathrm{ST}, 0}$ as the chiral fixed point and that in $\Delta_{c}^{\mathrm{AA}, 0}$ as the collinear fixed point. Hence our nonperturbative results in agreement with the higher-loop analysis in RG provides a strong support for the existence of the chiral as well as collinear fixed point, and the latter, in particular, suggests the possibility of continuous chiral phase transition in QCD once the $U(1)_{A}$ is effectively restored. The low-lying spectra we obtained also predict the scaling behaviors of symmetry breaking effects from small mass of the quarks as well as the $U(1)_{A}$ breaking effects (e.g. instanton) near the chiral phase transition point. Note that, unlike the earlier prediction, our prediction for the $\Delta_{\mathrm{SS}} \simeq 1.52(5)$ significantly differs from $\Delta_{\mathrm{S}} \simeq 1.67(1)$ of the $O(4)$ Heisenberg universality class and could offer a clue for judging $U(1)_{A}$ restoration scenario itself in the future lattice and experimental studies of the correlation length.

\section{Discussions}

In this proceeding we have reported our conformal bootstrap program for $O(n) \times O(2)$-symmetric CFTs with 4 [26]. As in $n \gg m=3$ case carried out in [27], we have observed singular behaviors in the bounds of the dimension of operators in different sectors. Although our identification for them to be actual CFTs is still phenomenological based on the past experiences, their agreement with earlier higher-loop perturbative results is a striking evidence for the validity of both methods. We, therefore, believe that the results in [9][10][12] together with ours are robust enough to conclude that the chiral phase transition in QCD, if being inside an attractor region in its RG flow, should exhibit continuous phase transitions with the critical exponents that we most precisely predict from the output of the conformal bootstrap program.

We end with several challenges to the conformal bootstrap program. Firstly, we had to resort to the earlier RG results to determine the symmetry breaking pattern. To be fully self-contained, it is imperative to know the signs of some OPE coefficients, but this is impossible from the bootstrap of the single correlator alone since all the OPE coefficients appear as the squares of them. The mixed- 
correlator bootstrap [47] with the energy-momentum tensor will fix the sign issue. However, the independent structures in the four-point function of the energy-momentum tensors are numerous [48], and the computational task would be even more intensive. Secondly we are urged to understand the meaning of kinks, for which the recently observed "spectrum jumping" behavior might be helpful [24]. It has also been observed in [47] that the simultaneous consideration of several correlators and assumptions on the number of relevant operators in the spectrum is so restrictive that it singles out the region around the kink (where the $d=3$ Ising model is supposed to live) as an isolated "island" in the space of entire $\mathbb{Z}_{2}$-symmetric CFTs. Our conclusion would become more convincing if we observed similar phenomena also in our models. All in all, it is quite plausible that deeper knowledge of the conformal bootstrap program will revolutionalize our understanding of the world. Then the resolutions of the controversies in QCD would be just a small example.

\section{References}

[1] J. L. Cardy, Scaling and renormalization in statistical physics. Cambridge University Press, 1996.

[2] A. Pelissetto and E. Vicari, "Critical phenomena and renormalization group theory," Phys.Rept. 368 (2002) 549-727, arXiv: cond-mat/0012164 [cond-mat]

[3] HotQCD Collaboration Collaboration, A. Bazavov et al., "The chiral transition and $U(1)_{A}$ symmetry restoration from lattice QCD using Domain Wall Fermions," Phys.Rev. D86 (2012) 094503. arXiv:1205.3535 [hep-lat]

[4] S. Aoki, H. Fukaya, and Y. Taniguchi, "Chiral symmetry restoration, eigenvalue density of Dirac operator and axial U(1) anomaly at finite temperature," Phys.Rev. D86 (2012) 114512 arXiv:1209.2061 [hep-lat]

[5] G. Cossu, S. Aoki, H. Fukaya, S. Hashimoto, T. Kaneko, et al., "Finite temperature study of the axial $\mathrm{U}$ (1) symmetry on the lattice with overlap fermion formulation," Phys.Rev. D87 no. 11, (2013) 114514 arXiv:1304.6145 [hep-lat]

[6] M. I. Buchoff, M. Cheng, N. H. Christ, H. T. Ding, C. Jung, et al., "The QCD chiral transition, Ua symmetry and the Dirac spectrum using domain wall fermions," Phys.Rev. D89 (2014) 054514 arXiv:1309.4149 [hep-lat]

[7] A. Pelissetto and E. Vicari, "Relevance of the axial anomaly at the finite-temperature chiral transition in QCD," Phys.Rev. D88 no. 10, (2013) 105018 arXiv:1309.5446 [hep-lat]

[8] K. I. Ishikawa, Y. Iwasaki, Y. Nakayama, and T. Yoshie, "Global Structure of Conformal Theories in the SU(3) Gauge Theory," Phys.Rev. D89(2014) 114503, arXiv:1310.5049 [hep-lat]

[9] A. Pelissetto, P. Rossi, and E. Vicari, "The Critical behavior of frustrated spin models with noncollinear order," Phys.Rev. B63 (2001) 140414 arXiv:cond-mat/0007389 [cond-mat]

[10] P. Calabrese and P. Parruccini, "Five loop epsilon expansion for $\mathrm{O}(\mathrm{n}) \mathrm{x} \mathrm{O}(\mathrm{m})$ spin models," Nucl.Phys. B679(2004) 568-596, arXiv: cond-mat/0308037 [cond-mat]

[11] P. Calabrese, P. Parruccini, and A. Sokolov, "Chiral phase transitions: Focus driven critical behavior in systems with planar and vector ordering," Phys.Rev. B66 (2002) 180403 arXiv: cond-mat/0205046 [cond-mat]

[12] P. Calabrese, P. Parruccini, A. Pelissetto, and E. Vicari, "Critical behavior of $\mathrm{O}(2) \times \mathrm{O}(\mathrm{N})$ symmetric models," Phys.Rev. B70(2004) 174439, arXiv: cond-mat/0405667 [cond-mat] 
[13] B. Delamotte, M. Dudka, Y. Holovatch, and D. Mouhanna, "About the relevance of the fixed dimension perturbative approach to frustrated magnets in two and three dimensions," Phys.Rev. B82 (2010) 104432 arXiv:1009.1492 [cond-mat.stat-mech]

[14] M. Tissier, B. Delamotte, and D. Mouhanna, "Heisenberg frustrated magnets: A Nonperturbative approach," Phys.Rev.Lett. 84 (2000) 5208-5211, arXiv: cond-mat/0001350 [cond-mat]

[15] M. Tissier, B. Delamotte, and D. Mouhanna, "XY frustrated systems: Continuous exponents in discontinuous phase transitions," Phys.Rev. B67 (2003) 134422 arXiv: cond-mat/0107183 [cond-mat]

[16] R. Rattazzi, V. S. Rychkov, E. Tonni, and A. Vichi, "Bounding scalar operator dimensions in 4D CFT,"JHEP 0812 (2008) 031 arXiv:0807.0004 [hep-th]

[17] V. S. Rychkov and A. Vichi, "Universal Constraints on Conformal Operator Dimensions,"Phys.Rev. D80 (2009) 045006 arXiv:0905.2211 [hep-th]

[18] F. Caracciolo and V. S. Rychkov, "Rigorous Limits on the Interaction Strength in Quantum Field Theory," Phys.Rev. D81 (2010) 085037, arXiv:0912.2726 [hep-th]

[19] D. Poland and D. Simmons-Duffin, "Bounds on 4D Conformal and Superconformal Field Theories," JHEP 1105 (2011) 017 arXiv: 1009.2087 [hep-th]

[20] R. Rattazzi, S. Rychkov, and A. Vichi, "Central Charge Bounds in 4D Conformal Field Theory," Phys.Rev. D83 (2011) 046011 arXiv:1009.2725 [hep-th]

[21] D. Poland, D. Simmons-Duffin, and A. Vichi, "Carving Out the Space of 4D CFTs," JHEP 1205 (2012) 110 arXiv:1109.5176 [hep-th]

[22] S. El-Showk, M. F. Paulos, D. Poland, S. Rychkov, D. Simmons-Duffin, and A. Vichi, "Solving the 3D Ising Model with the Conformal Bootstrap,"Phys.Rev. D86 (2012) 025022 arXiv:1203.6064 [hep-th]

[23] S. El-Showk, M. Paulos, D. Poland, S. Rychkov, D. Simmons-Duffin, and A. Vichi, "Conformal Field Theories in Fractional Dimensions," Phys.Rev.Lett. 112 (2014) 141601, arXiv:1309.5089 [hep-th]

[24] S. El-Showk, M. F. Paulos, D. Poland, S. Rychkov, D. Simmons-Duffin, and A. Vichi, "Solving the 3d Ising Model with the Conformal Bootstrap II. c-Minimization and Precise Critical Exponents," J.Stat.Phys. 157 (2014) 869, arXiv:1403.4545 [hep-th]

[25] F. Kos, D. Poland, and D. Simmons-Duffin, "Bootstrapping the $O(N)$ vector models," JHEP 1406 (2014) 091,arXiv:1307.6856.

[26] Y. Nakayama and T. Ohtsuki, "Bootstrapping phase transitions in QCD and frustrated spin systems," Phys. Rev. D 91, no. 2, 021901 (2015) [arXiv:1407.6195 [hep-th]].

[27] Y. Nakayama and T. Ohtsuki, "Approaching conformal window of $O(n) \times O(m)$ symmetric Landau-Ginzburg models from conformal bootstrap," Phys.Rev. D89(2014) 126009 arXiv:1404.0489 [hep-th]

[28] M. Hogervorst, H. Osborn, and S. Rychkov, "Diagonal Limit for Conformal Blocks in $d$ Dimensions," JHEP 1308 (2013) 014 arXiv: 1305.1321

[29] M. Yamashita, K. Fujisawa, M. Fukuda, K. Nakata, and M. Nakata, “A high-performance software package for semidefinite programs: SDPA 7,".

[30] “Sdpa official page.”. 
[31] P. Calabrese, A. Pelissetto, and E. Vicari, "Multicritical behavior in frustrated spin systems with noncollinear order,"Nucl.Phys. B709 (2005) 550-577 arXiv: cond-mat/0408130 [cond-mat]

[32] S. El-Showk and M. F. Paulos, "Bootstrapping Conformal Field Theories with the Extremal Functional Method," Phys.Rev.Lett. 111 no. 24, (2013) 241601, arXiv:1211.2810 [hep-th]

[33] R. D. Pisarski and F. Wilczek, "Remarks on the Chiral Phase Transition in Chromodynamics," Phys.Rev. D29 (1984) 338-341

[34] M. Grahl and D. H. Rischke, "Functional renormalization group study of the two-flavor linear sigma model in the presence of the axial anomaly," Phys.Rev. D88 no. 5, (2013) 056014. arXiv:1307.2184

[35] T. Sato and N. Yamada, "More about vacuum structure of Linear Sigma Model," PoS LATTICE2013 (2013) 430, arXiv:1311.4621 [hep-lat]

[36] P. Calabrese, P. Parruccini, and A. I. Sokolov, "Critical thermodynamics of a three-dimensional chiral model for N>=3," 68 no. 9, (Sept., 2003) 094415, Cond-mat/0304154

[37] M. Kindermann and C. Wetterich, "Phase transitions in liquid helium 3," Phys.Rev.Lett. 86 (2001) 1034-1037 arXiv:cond-mat/0008332 [cond-mat]

[38] M. De Prato, A. Pelissetto, and E. Vicari, “The Normal to planar superfluid transition in He-3," Phys.Rev. B70 (2004) 214519, arXiv: cond-mat/0312362 [cond-mat]

[39] J. Berges, N. Tetradis, and C. Wetterich, “Coarse graining and first order phase transitions," Phys.Lett. B393 (1997) 387-394 arXiv: hep-ph/9610354 [hep-ph]

[40] J. Berges and C. Wetterich, "Equation of state and coarse grained free energy for matrix models," Nucl.Phys. B487 (1997) 675-720, arXiv: hep-th/9609019 [hep-th]

[41] K. Fukushima, K. Kamikado, and B. Klein, "Second-order and Fluctuation-induced First-order Phase Transitions with Functional Renormalization Group Equations," Phys.Rev. D83 (2011) 116005 arXiv:1010.6226 [hep-ph]

[42] F. Karsch, "Scaling of pseudocritical couplings in two flavor QCD," Phys.Rev. D49 (1994) 3791-3794 arXiv:hep-lat/9309022 [hep-lat]

[43] Y. Iwasaki, K. Kanaya, S. Kaya, and T. Yoshie, "Scaling of chiral order parameter in two flavor QCD," Phys.Rev.Lett. 78(1997) 179-182,arXiv: hep-lat/9609022 [hep-lat]

[44] CP-PACS Collaboration Collaboration, A. Ali Khan et al., "Phase structure and critical temperature of two flavor QCD with renormalization group improved gauge action and clover improved Wilson quark action,"Phys.Rev. D63 (2001) 034502, arXiv: hep-lat/0008011 [hep-lat]

[45] M. D’Elia, A. Di Giacomo, and C. Pica, “Two flavor QCD and confinement,"Phys.Rev. D72 (2005) 114510 arXiv:hep-lat/0503030 [hep-lat]

[46] S. Ejiri, F. Karsch, E. Laermann, C. Miao, S. Mukherjee, et al., "On the magnetic equation of state in (2+1)-flavor QCD," Phys.Rev. D80 (2009) 094505 arXiv:0909.5122 [hep-lat]

[47] F. Kos, D. Poland, and D. Simmons-Duffin, "Bootstrapping Mixed Correlators in the 3D Ising Model,"arXiv:1406.4858 [hep-th]

[48] A. Dymarsky, "On the four-point function of the stress-energy tensors in a CFT," arXiv:1311.4546 [hep-th] 